\title{
NEUTRON TIME-OF-FLIGHT TEXTURE ANALYSIS
}

\author{
K. Feldmann, M.Betzl \\ Central Institute for Nuclear Research Rossendorf, Academy of Sciences of \\ GDR, P.0.Box 19, Dresden, 8051, GDR \\ W.KLeinsteuber, K. Walther \\ Laboratory of Neutron Physics, Joint Institute for Nuclear Research Dubna, \\ Head Post office 79, Moscow, USSR
}

\section{INTRODUCTION}

Spectroscopic methods like X-ray or thermal neutron diffraction are mainly used for quantitative texture analysis. The low absorption of neutrons by the most of isotopes makes them well-suited for bulk texture investigations averaging all texture inhomogeneities, even in the case of relatively coarse grained materials. Complete pole figures (PFs) can be determined without special preparation techniques. Serious difficulties arise in the study of hydrogen containing materials because of the very large incoherent scattering cross-section of hydrogen.

At present, texture investigation facilities at various neutron sources are in operation. Different variants of the angle dispersive neutron diffraction at stationary reactors are widely used. In the previous years powerful pulsed neutron sources were put into operation $/ 1,2 /$. On this base the energy-dispersive neutron time-of-flight diffraction (TOFD) has been applied to solve texture problems, especially in the field of low crystal symmetry and multiple phase materials as described in $13,4 /$.

\section{TIME-OF-FLIGHT DIFFRACTION}

Diffraction is determined by the Bragg's Law

$$
\lambda=2 d_{h k l} \cdot \sin \gamma
$$

There are two possibilities to satisfy this equation for discrete lattice spacings leading to two different experimental techniques:

- The incident beam is monochromatic. The Bragg angle has to be varied (conventional angle dispersive method).

- A polychromatic beam is used at constant scattering angle consisting of a given wavelength (or energy) spectrum (TOFD).

Here, the TOFD technique is considered.

Since neither wavelength nor energy sensitive detectors are available the time required to fly through a certain distance is measured to determine the energy of a given neutron. In this case the emission time of the neutron must be known, i.e. pulsed beams have to be used. The relation between time of $f$ light $T$ and energy $E$ or wavelength is given by

$$
T=\left(L_{1}+L_{2}\right) \cdot \operatorname{SQRT}\left(2 \cdot m_{n} / E\right)=b \cdot\left(L_{1}+L_{2}\right) \cdot \lambda
$$




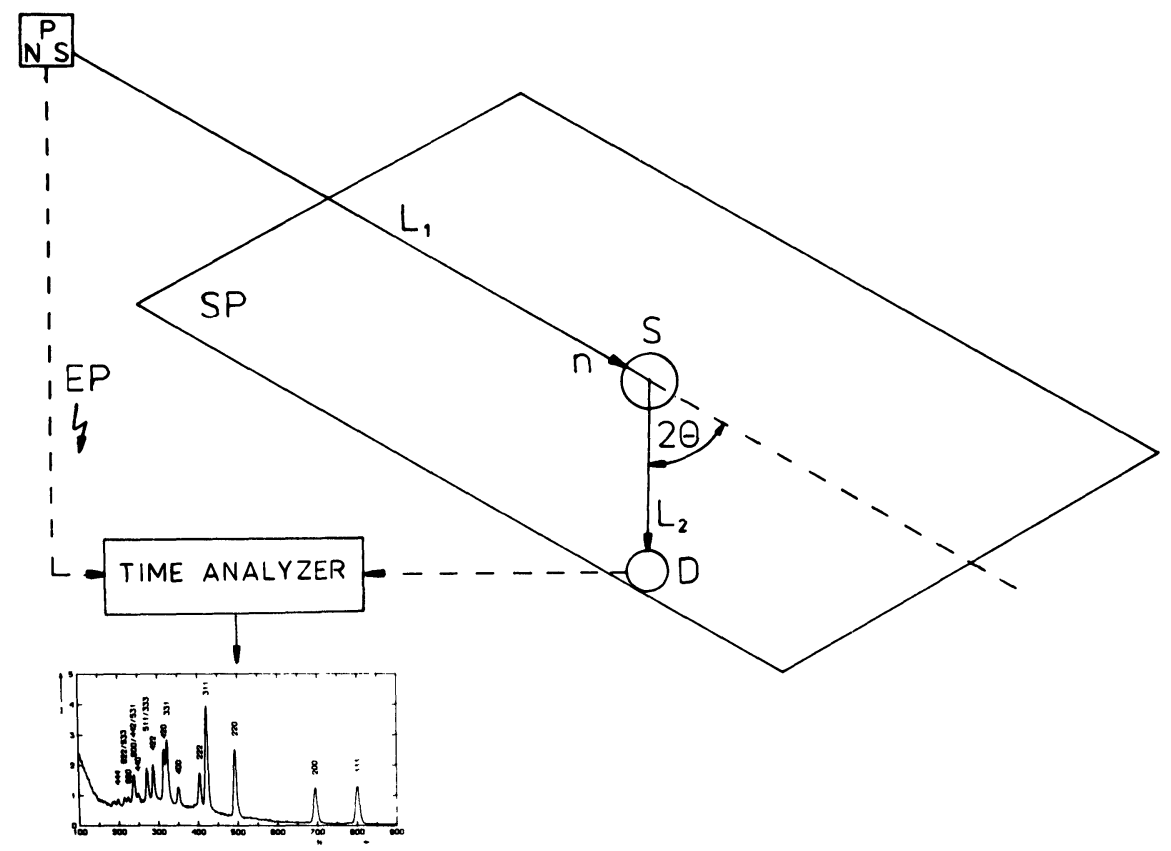

Figure 1 Layout of a TOFD experiment. The pulsed source (PNS) emits the neutron and the electronic start (EP) pulse. After flying through $L_{1}$ and $L_{2}$ and scattering in the sample $S$ the neutrons are recorded by the detector $D$. Storing the counter pulses in dependence on their arrival a TOFD spectrum is built up in the time analyzer.

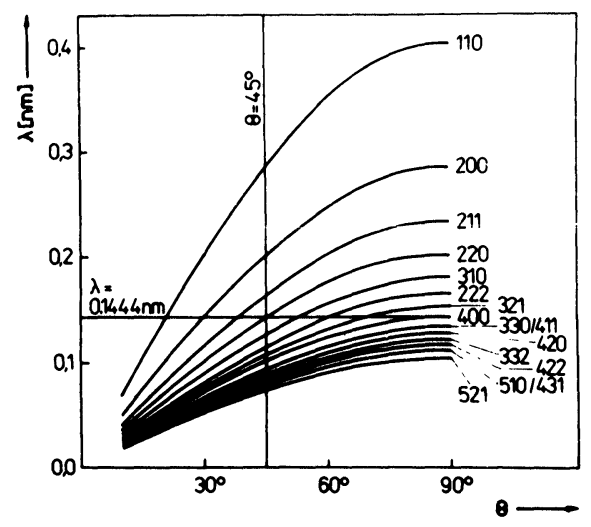

Figure 2 Bragg law for a bcc-lattice. The horizontal line represents the angle dispersive and the vertical one the TOFD experiment.

where $b=2.528 \cdot 10^{6} \mathrm{sec} / \mathrm{m}^{2}, m_{n}$ is the neutron mass and $L_{1}+L_{2}$ is the total flight path from the source via the ${ }^{n}$ specimen to the counter (see ${ }^{1}$ Fig.1). 
In TOFD the complete Bragg pattern, (all non-forbidden Bragg reflections) is measured simultaneously at constant scattering geometry as represented in Fig. 2 by the vertical line. In conventional technique the number of measurable reflections is limited by the wavelength. The resolution of TOFD is best for large Bragg angles. It is essentially determined by the pulse width. The separability of neighbouring peaks becomes better for longer flight paths.

\section{TOFD TEXTURE ANALYSIS}

In the TOFD as in conventional neutronographic studies also complete PFs can be determined. Eulerian cradles or triple axis goniometers having one vertical and two mutually perpendicular horizontal axes are used for PF scanning. The point mesh may be an equal area net, an equal angle or even any other one.

In the TOFD PFs from all reflections are measured simultaneously by only one scan. So, the experimental expense is nearly independent of the required quantity of PFs, i.e. the TOFD becomes more efficient, if the number of necessary PFs increases. Consequently, the method is especially suitable for preferred orientation studies in samples having low lattice symmetry or in multiple phase materials.

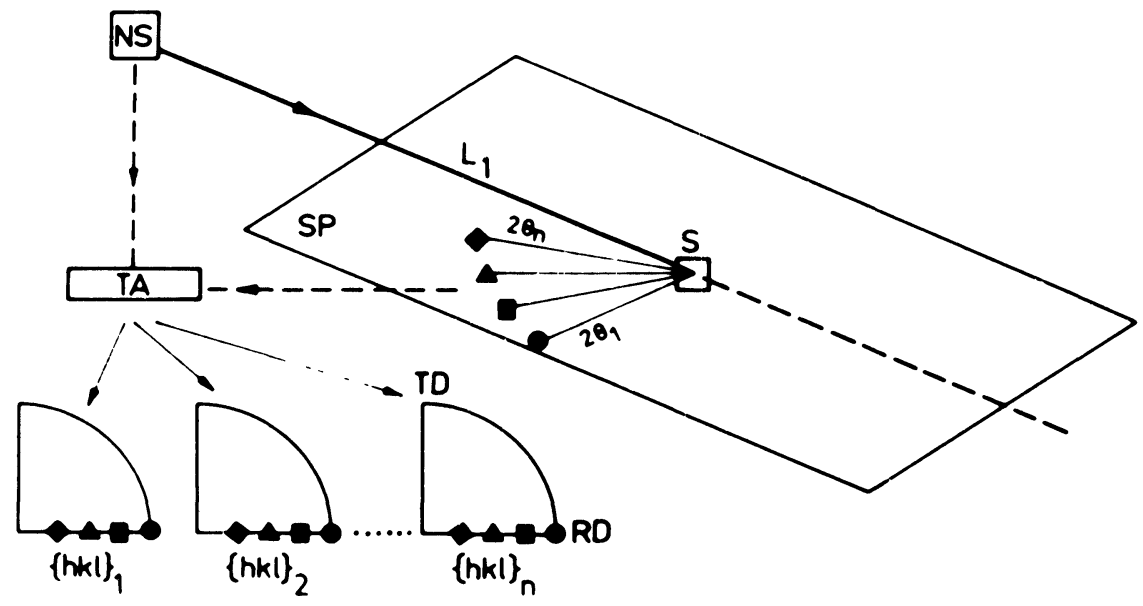

Figure 3 Application of a horizontal multidetector and the resulting distribution of points in the PFs.

Every TOFD spectrum is equivalent to the inverse PF of the corresponding specimen position. Unfortunately, the non-uniform point net on the inverse PF area determined by nature complicates the quantitative texture analysis on this base. The method is permits to observe the developement of texture components in real time processes.

Pole densities are determined via line profile analyses of Bragg reflections. The information of overlapped peaks may be used. The number of separable PFs is restricted by the spectrometer resolution, the efficiency of the available fit program and the lattice symmetry of the sample under investigation. To perform the line profile analysis the diffraction patterns have to be normalized with respect to wavelength independent neutron flux. The wavelength distribution of the incoming beam is reflected by the spectrum of a pure incoherent scatterer like vanadium. 
The relatively long time (>10 minutes) required to record one spectrum (one point in all PFs) is a serious drawback of the TOFD. For more efficiency multidetectors are used as shown in Fig.3. The counters are situated in a way to ensure constant tilt angle steps. The density of point meshs on the PFs may be diluted from the equator to the pole (similar to the equal area distribution). Another way to make the measurements more rapid is the use of a TOF multidetector arrangement equivalent to the Riso one $/ 5 /$ as discussed now $/ 6 /$.

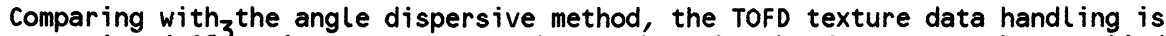
more expensive ( $>10^{3}$ points per spectrum). On the other hand, TOFD may be applied for a very wide range of preferred orientation problems.

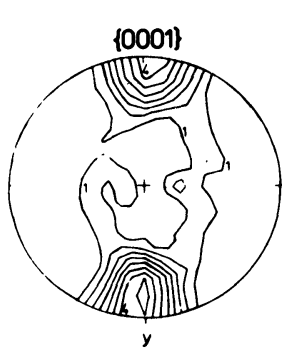

a
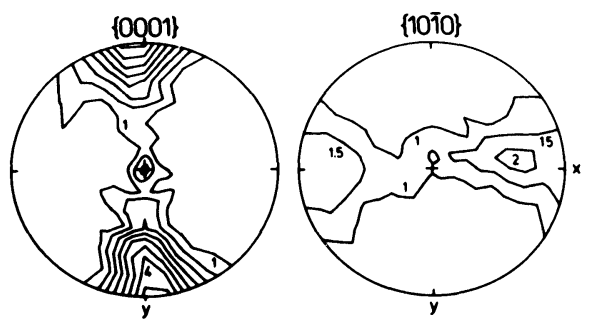

C

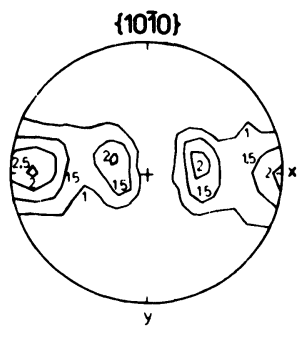

.
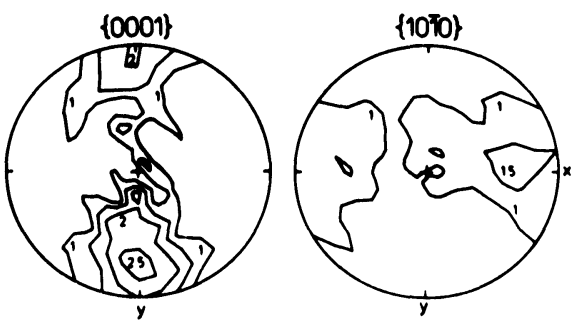

a

Figure 4 Basal and prism-I PFs of orthogneisses taken from the crest (a), the dipping flank $(b, c)$ and the limb (d) of the recumbent fold.

\section{APPLICATIONS}

The standard procedure in TOFD texture analysis performed at the NSWR texture diffractometer $/ 7 /$ at the IBR-2 pulsed reactor in Dubna is complete PFs measurement. Efforts were made to study the quartz partial texture in natural quartzitic rocks. In $18,9 /$ the quartz fabrics of gneisses of an asymmetric fold structure have been analyzed in dependence on their position in the rock complex. In Fig.4 the basal and prism-I PFs of the four samples are shown. All basal. PFs are characterized by point maxima at $y=a$ connected by ac-girdles. The texture sharpness increases from the limb to the crest of the fold. This is confirmed by prism PFs and can be understood taking into account the increasing deformation intensity. In the prism PFs a fabric rotation around the $y=a$ axis is found from the dipping $f$ lank to the crest of the fold. 


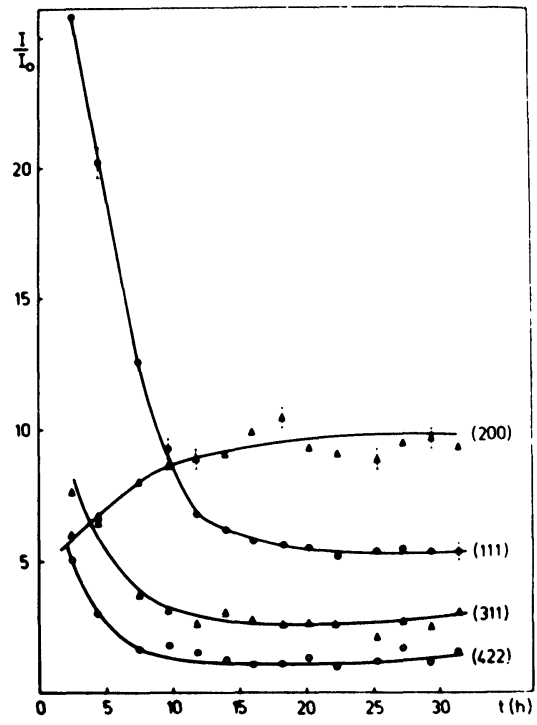

Figure 5 Intensity versus annealing time of four low index copper reflections.

The constant scattering geometry in TOFD is well-suited to observe texture component formations in real-time experiments. Fig.5 shows the behaviour of four Low index reflections of copper during the recrystallization process $110 /$. The exposition time of 30 minutes per spectrum enabled by low recrystallization temperature $(225 \mathrm{C})$ was too long to observe technically relevant processes. More promising times (<1min.) arereported in/11/ to observe the hydratation kinetics of $\mathrm{Ca}_{3} \mathrm{Al}_{2} \mathrm{O}_{6}$ at the IBR-2 reactor.

3 Texture data are extracted from TOFD by line profile analysis. The position, the maximum and the line width of the studied peak may be fitted. So, the method include information on residual stress distributions in the considered material. Special efforts have to be made to ensure adequate resolution and intensity parameters.

The magnetic moment of neutrons was tried to apply for the study of magnetic sublattice anisotropies in magnetically ordered materials $/ 12 /$. Two methods are available to separate magnetic from nuclear diffraction:

- The difference between various order PFs is expected to represent the pure magnetic texture component, because of the sin $V / \lambda$ dependence of the magnetic scattering. Up to now, careful measurements did not provide satisfactory results, probably due to extinction effects. The constant scattering geometry for different order PFs favours the TOFD for such type studies.

- The magnetic diffraction can be suppressed completely, if the specimen is magneti - zed up to its saturation with magnetization direction parallel to the scattering vector. The difference between field off and field on measurements represents the magnetic component. Of course, such experiments are not reproducible. 


\section{CONCLUSIONS}

Neutron TOFD is shown to be an efficient tool for bulk texture investigations. It is applicable for the most of materials with the exception of hydrogen containing substances, e.g. of polymers. The method permits to study a wide range of texture problems in metallurgy and metal physics as well as in petrofabric analysis. In comparision with the conventional technique it is especially suited to investigate low symmetric and multiple phase materials. The relatively long exposition times and the more complicated data handling due to profile analysis are restrictions of the method. The applications of multidetectors decreases the required time for measurements without any difficulties in PF scanning.

Very short exposition times in TOF method reported may be promising to observe the kinetics of texture formation immediately, caused by external influences.

Magnetic texture studies are confronted by methodical difficulties up to now. Further progress will, however, be made in future.

\section{REFERENCES}

1. V.D.Ananiev et al., Proc. Int.Conf. on Neutron Scattering in the Nineties (IAEA, Vienna, 1985), p.63

2. A.J.Leadbetter,Proc.Int.Conf.on Neutron Scattering in the Nineties (IAEA, Vienna, 1985) p.219.

3. K.Feldmann, Proc. Workshop on Experimental Techniques of Texture Analysis. (Ed. H.J.Bunge, Oberursel, DGM Informationsgesell (schaft 1986), p.253.

4. K.Feldmann, Textures and Microstructures, 10, 309 (1989)

5. D.Juul-Jensen, D., Proc. Workshop on Experimental Techniques of Texture Analysis (Ed.H.J.Bunge Oberursel, DGM Informationsgesel(schaft, 1986), p.217

6. K.Walther, this volume.

7. B.N.Ananiev et al., JINR Communication P14-84-827, Dubna 1984, in Russian

8. L.P.Drechsler et al., Textures and Microstructures, 8-9,737 (1988)

9. V.Damm et al., Textures and Microstructures, 12, 15 (1990)

10. W. Matz and K. Feldmann, JINR Communication P14-82-265, Dubna 1982, in Russian. 11. A.Balagurov and G.Mironova, JINR Short Communication N19-86, Dubna 1986, in Russian

12. K.Hennig, J.Bouillot and A.Muecklich, Annual Report (ILL Grenoble, 1983) 